\title{
Insertion Materials for Batteries
}

Until 1871, with the serendipitous discovery of the dynamo by Gramme, electrochemical batteries were the sole sources of electrical current, from which important discoveries in physics were made. During the last decade, the use of portable electrical devices ranging from cordless tools to video cameras, laptop computers, and cellular telephones has led to a doubling every three years in the demand for batteries. The continuous need for smaller and lighter batteries able to accept rapid charging has called for extensive research into electrode materials, electrolytes and their associations.

A battery harnesses a chemical reaction to generate electrical energy. When two electrodes of different chemical potentials $\mu_{1}$ and $\mu_{2}$ are brought into contact through a common ionic conductor (the electrolyte), the electrons liberated by reduction and oxidation (redox) reactions can be used as electricity. As shown in Fig. 1 illustrating the basic principles of a battery, the ionic and electronic fluxes created by a redox reaction such as metal $\mathrm{M} \rightarrow \mathrm{M}^{+}+\mathrm{e}^{-}$occuring in both electrodes are decoupled: the ion flow goes from electrode 1 to electrode 2 inside the battery via the electrolyte whereas the electron flow outside the battery driven by the current collectors delivers electricity to the load.

The electromotive force (EMF) is defined as $E_{1,2}=1 / F\left(\mu_{1, M}-\mu_{2, M}\right)$ where $F$ is Faraday's constant and the potentials $\mu$ refer to metal $M$ in the electrodes. In this scheme, both electrodes comprise an interface at which ion/electron interchange takes place when a chemical potential is applied. There are several ways for realising these conditions. Having the metal in contact with an electrolyte containing $\mathrm{M}^{+}$is the simplest case (Type I electrode) where the chemical source of $\mathrm{M}$ is the electrolyte itself, possible through local dissolution and transport processes in the vicinity of the interface.

However, the most elegant and general approach is to use a material possessing

Christiane Poinsignon is a Director of Research at the Laboratoire d'lonique et d'Electrochimie des Solides - Grenoble (LIES-G), BP 75, F-38402 St. Martin d'Hères. After graduating from Nancy University in 1967, she joined the French CNRS in 1971 and was awarded a Ph.D. from the Politecnicum Institute of Lorraine in 1977. She was detached to the Institut Laue-Langevin, Grenoble, in 1980-86.

Michel Armand graduated from the ENS, St. Cloud, in 1970 and then spent two years as a research assistant at Stanford in the USA before joining the CNRS. He received his Ph.D. in electrochemistry in 1978 and has been a Director of Research at LIES-G since 1982. He serves on the editorial boards of Solid-State Ionics, J. Power Sources, J. Appl. Electrochem., and Bull. Electrochem.

\section{PRINCIPLES AND DEVELOPMENT}

\author{
C. Poinsignon, M.B. Armand
}

Laboratoire d'lonique et d'Electrochimie du Solide

Grenoble, France

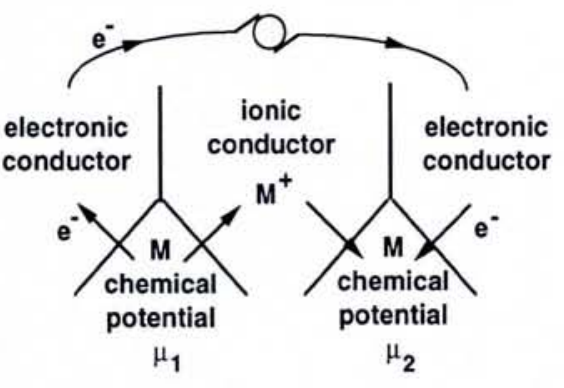

Fig. 1 - Schematic representation of the principle of an insertion-type storage battery. The insertion material combines the three essential functions of an electrode (electronic and ionic conductivity; source of a chemical potential $\left.\mu_{M}\right)$.

recorded in a short time ( 3 to 10 minutes) while the electrode potential is controlled by a digitized electrochemical device which simultaneously records, as a function of time, the electrical charge exchanged by the electrode material.

Other major improvements in battery per-

intrinsically the three functions of the electrode namely, electronic and ionic conductivity (mixed conductivity) and a source of chemical potential $\mu_{M}$. These properties define what is called an insertion material [1] - a concept which has proved to be a major advance in electrochemistry and provides considerable momentum for research and development worldwide.

\section{Improved Performance}

Major improvements in the performance of batteries based on insertion materials can be realised by understanding structural changes that occur in the materials during charging and discharging. Real-time investigations at the atomic level lead to the direct observation of modifications and phase changes which may impede the reversibility of the redox process. Electrode reactions can be monitored using in situ techniques such as x-ray and neutron diffraction, NMR, and optical spectroscopies (IR, UV, visible, Raman) as well as by voltamperometry [2].

The best information about the structure of insertion materials and their modification during the insertion and de-insertion of ions into the insertion material is undoubtedly gained by coupling these techniques. For example, a powerful method combines controlled scanning potential chronoamperometry with real-time powder neutron diffraction. Neutron diffraction benefits from the low interaction of neutrons with matter, and the different transparencies of materials for neutrons to study an electrode with dimensions approaching those found in commercial batteries. Furthermore, the use of a positionsensitive detector with a large angular aperture $\left(80^{\circ}\right.$ on the D1B powder diffractometer at the Institut Laue-Langevin's High Flux Reactor neutron source, with an increase to $160^{\circ}$ foreseen) allows a diffractogram to be formance based on insertion materials can be achieved using novel technologies, notably electrochemistry in thin films to more effectively counter intrinsic limitations due to mass transport, either in electrolytes (conductivity) or electrode materials (diffusion). High-power batteries for electric vehicles using polymer electrolytes are the best illustration of high energy density batteries built from thin $(100 \mu \mathrm{m})$ stacked units (Fig. 2). Using the fundamental laws of thermodynamics, the specific power $P$ of such a battery can be expressed as:

$$
\partial P / \partial V=\bar{E}^{2} \eta(1-\eta) \sigma / d^{2}
$$

for a volume $V$, average EMF $\bar{E}$, energy efficiency (the ratio of the output to input energy) $\eta$, conductivity $\sigma$, and overall thickness over which mass transport takes place equal to $d$. The formula indicates the importance of reducing $d$ to obtain high power densities.

\section{Thermodynamics}

The electrode potential $E$ at a given temperature $T$, pressure $P$ and concentration $n$ of atomic species $j$ and $k$ is defined as

$$
E=1 / F \mu_{M}
$$

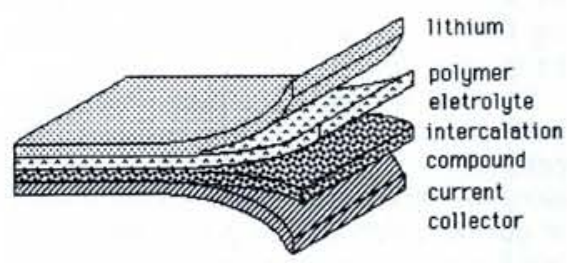

Fig. 2 - A schematic drawing of a lithium thin-film battery with a polymeric electrolyte. 
where the potential is given by

$$
\mu=\left(\mathrm{d} G / \mathrm{d} n_{\mathrm{j}}\right)_{n_{\mathrm{k}}}={ }_{n_{\mathrm{j}}, T, P}
$$

for a free energy $G$. The potential of the metal $\mathrm{M}$ is essentially the sum of the chemical potential contributions for ions and electrons, i.e., $\mu_{\mathrm{M}}=\mu_{\mathrm{i}}+\mu_{\mathrm{e}}$. Fermi-Dirac statistics have to be used for both particles as the lattice of the insertion material possesses a fixed number of sites. Hence,

$$
\mu=\mu_{0}+(R T / F) \ln \text { ([occupied sites]/ }
$$

for a standard chemical potental $\mu_{\mathrm{o}}$. The site occupancy $y$ is defined as $y=x / x_{\max }$ where $x$ is the number of inserted species per unit of formula, as in $\mathrm{H}_{x} \mathrm{MnO}_{2}$, and $x_{\max }$ is the maximum value of $x$. This thermodynamic approach is implied in Nernst's law used for ions in electrochemistry. It gives in the case of insertion materials the potential of the insertion electrode, assuming that all insertion sites are energetically equivalent.

For electrons, one can define the total band occupancy $y_{\mathrm{e}}$ as

$$
y_{\mathrm{e}}=\int_{\varepsilon}^{\varepsilon+L} \frac{D(P) P(\varepsilon) \mathrm{d} \varepsilon}{D(\varepsilon) \mathrm{d} \varepsilon}
$$

for a Fermi distribution $P(\varepsilon)$ and density of states $D(\varepsilon)$ of energy $\varepsilon$. Assuming the band is narrow, the expression can be simplified if the band width $L=0$, whereupon $D(\varepsilon)$ is a Dirac function. The electron chemical potential which is equal, at equilibrium, to the Fermi energy then becomes:

$$
\mu=\mu_{\mathrm{o}}+(R T / F) \ln \left(y_{\mathrm{e}} /\left[1-y_{\mathrm{e}}\right]\right) .
$$

Applying Eqs. 1, 2 and 3 to both ions and electrons results in:

$$
\begin{aligned}
E=E_{0} & +(R T / F)\left(\ln \left[y_{i} /\left\{1-y_{i}\right\}\right]\right. \\
& \left.+\ln \left[y_{\mathrm{e}} /\left\{1-y_{\mathrm{e}}\right\}\right]\right) .
\end{aligned}
$$

The equation implies two possibilities:

a) one type of site is limiting -

$$
\text { or } \begin{aligned}
& y_{\mathrm{i}}=y=x / x_{\max } \text { with } y_{\mathrm{e}} \ll<y_{\mathrm{i}} \\
& y_{\mathrm{e}}=y=x / x_{\max } \text { with } y_{\mathrm{i}} \ll<y_{\mathrm{e}} \text {; }
\end{aligned}
$$

b) both types of site are simultaneously limiting -

$$
y_{\mathrm{i}}=y_{\mathrm{e}}=y=x / x_{\max }
$$

and $\mathrm{E}=\mathrm{E}_{\mathrm{o}}+\left(\delta_{\mathrm{i}, \mathrm{e}} R T / F\right) \ln (y /[1-y])$

where the constant $\delta_{i, e}=1$ or 2 depending on a limitation either by ions or electrons or by both species. It is departures from theoretically predicted values for $E$ given by Eqs. 4 and 5 which encourage electrochemists to look for the origin of the deviations in terms of crystallographic observations.

\section{Manganese Dioxide Electrodes}

Manganese dioxide used as a cathode material in association with zinc is an essential component of commercial 1.5 volt, alkaline or Leclanché batteries based on $\mathrm{KOH}$ or $\mathrm{NH}_{4} \mathrm{Cl}$ electrolytes, respectively. $\mathrm{MnO}_{2}$ exists as numerous isotypes [3]; the electrochemically active form for proton insertion is known as disordered material. It is described by De Wolff [4] as a regular intergrowth of ramsdellite and pryolusite. Initially considered as an example of a perfectly non-stoichiometric, Type I electrode material [1], the structure of $\mathrm{MnO}_{2}$ remained an open question. It is defined by the intercalation reac-

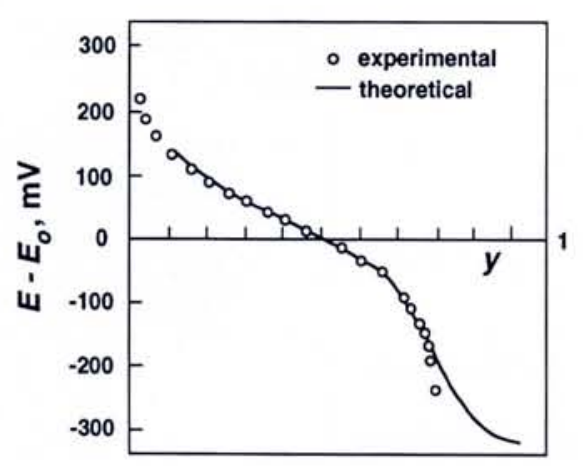

cal Manganese Dioxide - CMD ) was different to that for electrochemically produced material $\left(\varepsilon-\mathrm{MnO}_{2}\right.$ or Electrochemical Manganese Dioxide - EMD). The problem was that the $\gamma$ - and $\varepsilon-\mathrm{MnO}_{2}$ structures were not fully understood, along with details of the reduction mechanisms in spite of the reaction being fairly straightforward. In particular, there was no efficient method of characterising industrial samples of CMD and EMD, and thus no means to relate atomicscale arrangements in these materials to their behaviour in batteries. It eventually became clear that structural or textural transformations apparently take place at proton insertions above 0.25 electrons per $\mathrm{Mn}$ atom, whereupon the insertion process becomes irreversible, which means that batteries based on this system are not rechargeable.

\section{Crystallochemical diagram}

Structural characterisation of $\gamma$ - and $\varepsilon$ tion which expresses the reduction of manganese dioxide in oxyhydroxide according to the simple reaction [2]:

$$
\mathrm{MnO}_{2}+x \mathrm{H}^{+}+\mathrm{e}^{-} \rightarrow \mathrm{H}_{x} \mathrm{MnO}_{2}
$$

for which values of the coefficient $x$ can vary from 0 to the maximum value $x_{\max }$. The oxhydride thus constitutes a solid solution electrode as defined by Steele [5].

The main property of a Type I compound is the presence of a reversible electrochemical reaction in which transport of matter involves fluxes of charged species $\left(\mathrm{M}^{+}\right.$and $\left.\mathrm{e}^{-}\right)$in the bulk of the material whatever the composition. Insertion of $\mathrm{H}^{+}$in $\mathrm{MnO}_{2}$ was assumed for a long time to arise in a homogeneous phase with all insertion sites being energetically equivalent. Under these circumstances, the potential of the insertion electrode should vary according to a generalisation of Nernst's law

$$
\begin{array}{r}
E=E_{\mathrm{o}}+R T \ln \text { ([oxidised species]/ } \\
\text { [reduced species]) }
\end{array}
$$

where the oxidation/reduction ratio is expressed by the the insertion ratio $y=x / x_{\max }$. The theoretically predicted behaviour is given by

$$
\begin{gathered}
E=E_{\mathrm{O}}-2.3 R T / F[\mathrm{pH}] \\
+(R T / F) \ln (y /[1-y]) .
\end{gathered}
$$

where $[\mathrm{pH}]$ is the $\mathrm{pH}$ of the electrolyte. The potential calculated using this expression decreases regularly as a function of $y$ and is always greater than the measured one (see Fig. 3). Numerous electrochemists have confirmed that the variation of $E(y)$ does not follow the predicted behaviour [6].

Eq. 6 is in fact limited to a low rate of reduction [6] so the the assumption of a homogeneous phase reduction has been widely contested $[7,8]$. Using the De Wolff's results [4]. Atlung was the first to propose a new approach, namely a two-step reaction involving a transformation of $\mathrm{MnO}_{2}$ to $\mathrm{MnOOH}$ via $\mathrm{H}_{0.5} \mathrm{MnO}_{2}$ [8-11]. However, it proved impossible to accurately fit the theoretical and experimental variations of $E$ with the insertion ratio $y$ and to explain the observed behaviour for $y>0.5$. Moreover, the behaviour of $\mathrm{MnO}_{2}$ produced using chemical techniques $\left(\gamma-\mathrm{MnO}_{2}\right.$ or so-called Chemi-
$\mathrm{MnO}_{2}$ was clearly needed before the reduccrystallography of the two compounds is based on an ideal ramsdellite model affected by two kinds of defects, namely: a) stacking disorder comprising a partially random interspersing of pyrolusite (rutile isotype) structural elements with a ramsdellite structure (De Wolff disorder [11]); and b) microtwinning of the ramsdellite structure on the (021) and/or (061) growth planes. This model allows a numerical simulation of the diffraction data (both $x$-ray and neutron), and by comparing with experimental data, gives access for the first time to structural parameters describing $\mathrm{CMD}$ and $\mathrm{EMD}$ materials. The same approach can also be applied to the two $\mathrm{MnOOH}$ oxyhydrides known as groutite and manganite, topologically related to ramsdellite and pyrolusite, which appear during the reduction of manganese dioxides in protonic media. Hence, the proton- $\mathrm{MnO}_{2}$ system can in principle be completely described by the crystallochemical diagram shown schematically in Fig. 4 .

\section{Neutron diffraction}

The validity of the structural model has been verified by recording in situ the neutron diffraction pattern of a commercial sample of $\mathrm{MnO}_{2}$ (Sedema WSA) during electrochemical reduction in a $7 \mathrm{~N}$ solution of $K O D$ in $\mathrm{D}_{2} \mathrm{O}$, where $\mathrm{KOD}$ is used in place of $\mathrm{KOH}$ to suppress the background due to the large cross-section of protons for incoherent scattering. The evolution of the diffraction patterns with the degree of intercalation/reduction (Fig. 5) mainly shows: a) a shift of the diffraction lines; b) a rapid shift of the (021) and (121) lines at about 0.5 D per $\mathrm{Mn}$; and c) the appearance of characteristic lines from $M n(O D)_{2}$ (pyrochroïte) owing to the disproportionation of $\mathrm{D}_{x} \mathrm{MnO}_{2}$.

The results support the reduction process suggested by Maskell [9] for $\mathrm{MnO}_{2}$ in a $1 \mathrm{~N}$ aqueous solution of potassium hydroxide. Proton insertion in the $1 \times 2$ channels of the ramsdellite (see Fig. 4) takes place in two steps corresponding to two different hydrogen bonding geometries below and above the critical $0.5 \mathrm{H}$ per $\mathrm{Mn}$ composition. Protonic attack of the pyramidal oxygen atoms of the ramsdellite chain forms locally elements tion mechanism could be understood. The 


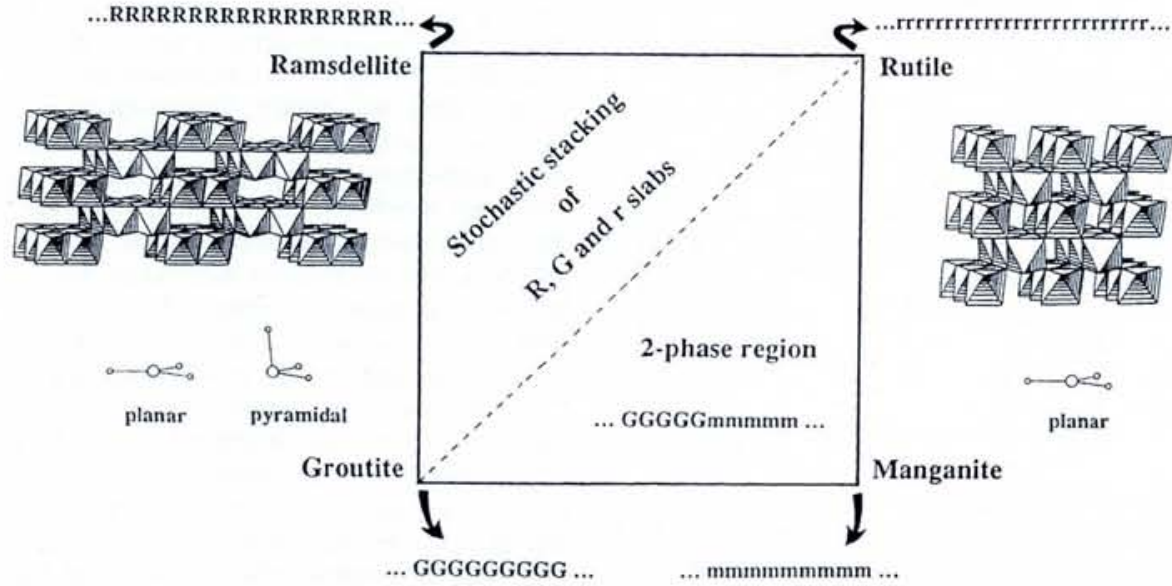

Fig. 4 - Structural phase diagram of the $\mathrm{MnO}_{2}-\mathrm{MnOOH}$ system. Ramsdellite (orthorhombic) and pyrolusite or $\beta-\mathrm{MnO}_{2}$ (tetragonal, isotype of rutile) are stoichiometric isotypes of $\mathrm{MnO}_{2}$, pyrolusite is the more stable. The reaction products are groutite and manganite, respectively, and both are described by the same arrangement of $\mathrm{MnO}_{2}$ octahedra along the a and c crystallographic directions. In the b-direction, ramsdellite exibits rows of two full octahedra and two empty, and pyrolusite one full and one empty, defining $1 \times 2$ and $1 \times 1$ channels, respectively. $\gamma$ $\mathrm{MnO}_{2}$ is a ramsdellite network with pyrolusite slabs interspersed (described as stacking disorder); $\varepsilon-\mathrm{MnO}_{2}$ represents microtwinning of the $\gamma$-structure. Stochastic stacking of ramsdellite $R$, pyrolsute $r$, and groutite $G$ slabs can only exist in the upper part of the diagram, for a certain reduction rate; phase separation occurs in the lower part.

of the groutite structure. When the ramsdellite chains are completely reduced, the $\mathrm{H} / \mathrm{Mn}$ ratio equals $1-\alpha$ where $\alpha=P_{y} /\left(2-P_{y}\right)$ represents the ratio of proton sites available in the pryolusite slabs to the total number of proton sites in the $\gamma$ - or $\varepsilon-\mathrm{MnO}_{2}(0.05<<0.2$ depending on the the sample) and $P_{Y}$ is the number of pyrolusite slabs. Above this composition, proton attack of the oxygen atoms in the planes of the crystal structure leads to a partial destruction of the structure in concentrated KOD electrolytes.

The results of neutron diffraction experiments show that the first step of the intercalation does not strongly affect the topology of the lattice so this step should be reversible. The appearance of pyrochroïte at the end of the intercalation process illustrates the irreversibility of this system for reductions above $1-\alpha$. The reversibility for reduction between these two limits only depends on the proportion of interspersed ramsdellite and pyrolusite blocks. The electrochemically reversible variety of manganese dioxide isotype suitable for proton insertion therefore appears to be ramsdellite. It is this material which should be used as cathode materials for rechargeable (so-called secondary) as opposed to expendable (primary) batteries.

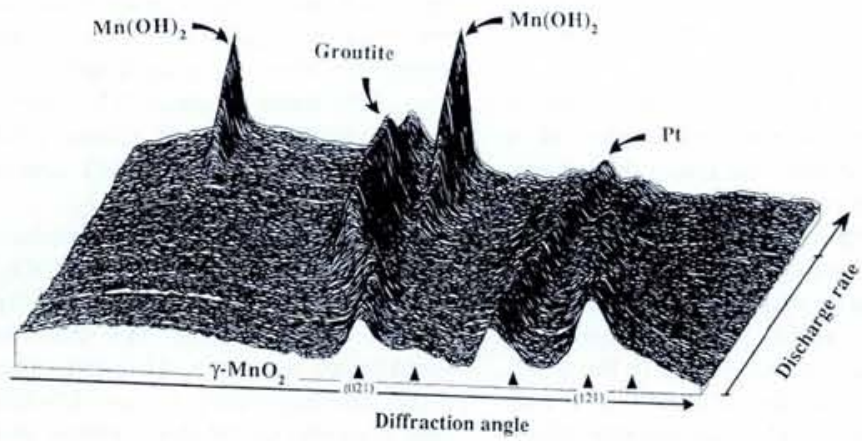

Fig. 5 - Evolution of the neutron diffraction pattern with the degree of insertion during in situ reduction (discharge) of $\gamma-\mathrm{MnO}_{2}$ (Sedema WSA) showing a rapid shift in the (021) and (121) peaks at about $0.5 \mathrm{D}$ per $\mathrm{Mn}$ and the appearance of $\mathrm{Mn}(O D)_{2}$ peaks. rôle in controlling the rate of hydrogen insertion and de-insertion and in minimizing the reduction in charging capacity. Our own results obtained by chronoamperometric studies on $\mathrm{LaNi}_{5-y} \mathrm{M}_{y} \mathrm{H}_{x}$ electrodes with $\mathrm{M}=$ $\mathrm{Al}, \mathrm{Mn}$, and $\mathrm{Co}$ and $y=0.5$ confirm earlier observations [14]: the full hydrogen capacity is de-inserted over potential ranges of $0.2 \mathrm{~V}$ in 80 hours for $\mathrm{M}=\mathrm{Al}$, of $0.130 \mathrm{~V}$ in 40 hours for $\mathrm{M}=\mathrm{Mn}$, and of $0.05 \mathrm{~V}$ in 30 hours for $\mathrm{M}=\mathrm{Co}$ (each value being for discharge by applying potential steps of $20 \mathrm{mV}$ every 10 hours).

Coupled techniques of real-time neutron diffraction and linear potential scan chronoamperometry have also been applied to an in situ study of the charging and discharging of a $\mathrm{LaNi}_{4.5} \mathrm{Al}_{0.5} \mathrm{D}_{x}$ electrode in a KOD-7N electrolyte in order to understand the effect of $\mathrm{Al}$ on the storage capacity and on phase stability during cycling [6]. The evolution of the $\alpha$ - and $\beta$-phase diffraction lines as a function of the rate of mass transfer associated with the controlled insertion/de-insertion of deuterium describes variations of cell parameters of the $\alpha$ - and $\beta$-phase domains present in the electrode [6]; the evolution also allows the deuterium de-insertion kinetics for both phases to be reliably estimated [14].

The formation of the isolated domains of the $\beta$-phase is likely to be responsible for the loss of capacity observed after repeated insertion/de-insertion cycles in $\mathrm{KOH}$. The time-resolved diffraction experiment is probably the only technique capable of providing direct information on insertion/de-insertion kinetics of both $\alpha$ - and $\beta$-phases during the charging/discharging of intermetallic hydride electrodes. However, further experiments are needed to elucidate the rôle of substitution for nickel on the kinetics.

[1] Armand M.B., in Materials for Advanced Batteries; Eds.: D.W. Murphy, J. Broadhead and B.C.H. Steele (Plenum, New York) 1979. [2] Thompson A.H., J. Electrochem. Soc. 126 (1979) 608; Chabre Y., idem. 138 (191) 329. [3] Ripert M. et al., Mater. Res. Soc. Proc. Series 210 (1991) 359.

[4] De Wolff P.E., Acta Crystallogr. 12 (1959) 341.

[5] Steele B., in Fast Ion Transport in Solids; Ed.: W. Van Gool (North-Holland, Amsterdam) 1973.

[6] Latroche M. et al., J. Alloy \& Compounds 189 (1992) 59.

[7] Gabano J.P. et al., Z. Phys. Chem. 46 (1965) 359; McBreen J., Electrochem. Acta 20 (1975) 221, J. Power Sources 5 (1975) 525.

[8] Atlung S. \& Jacobsen T., Electrochem. Acta 21 (1976) 575.

[9] Maskell W.C., Shaw J.A.E. \& Tye F.L., J. Appl. Electrochem. 12 (1982) 101.

[10] Atlung S. \& Jacobsen T., Electrochem. Acta 26 (1981) 144.

[11] Atlung S. \& West K., J. Power Sources 26 (1989) 139.

[12] Van Vucht J.H.N., Kuijpers F.A. \& Bruning H.C.A.M., Philips Res. Rep. 25 (1970) 133,1413 .

[13] Koh J.T. et al., J. Less-Common Metals 113 (1989) 89.

[14] Poinsignon C. \& Dalphrase N., Proc. Mater. Res. Soc. Fall Meeting, Boston, 1992 (to be published). 